\title{
Transtornos psicóticos associados ao período do climatério
}

\author{
Psychotic disorders associated with the climate period \\ Trastornos psicóticos asociados con el periodo climático
}

Recebido: 09/07/2021 | Revisado: 20/07/2021 | Aceito: 09/08/2021 | Publicado: 21/08/2021

\author{
Bruna Aguiar de Negreiros \\ ORCID: https://orcid.org/0000-0001-8278-4475 \\ Faculdade de Ciências Humanas, Exatas e Saúde do Piauí, Brasil \\ E-mail: brunanegreiross@gmail.com \\ Ysla Pontes Feitosa \\ ORCID: https://orcid.org/0000-0003-2571-88X \\ Faculdade de Ciências Humanas, Exatas e Saúde do Piauí, Brasil \\ E-mail: ysla99@outlook.com \\ Yasmin Clara Fernandes Ribeiro \\ ORCID: https://orcid.org/0000-0002-1443-733X \\ Faculdade de Ciências Humanas, Exatas e Saúde do Piauí, Brasil \\ E-mail: yasminclarafernandes37@gmail.com \\ José Lopes Pereira Junior \\ ORCID: https://orcid.org/0000-0001-9519-9363 \\ Universidade Federal do Piauí, Brasil \\ E-mail: josejrfarmaceutico@gmail.com
}

\begin{abstract}
Resumo
A menopausa é um período composto por diversas mudanças e transformações na vida da mulher, caracterizando o final do período reprodutivo dessas. Dessa forma, a diminuição significativa dos hormônios no organismo feminino resulta em um conjunto amplo de sinais e sintomas desagradáveis e característicos dessa fase, com destaque para manifestações psiquícas. O objetivo do trabalho consiste em realizar um levantamento bibliográfico acerca das desordens psiquiátricas manifestadas durante o período da menopausa. Realizou-se uma busca nas bases de dados Pubmed, SciELO, Lilacs e Cochrane Library com os descritores "Menopause" OR "Climacteric" OR "Middle Aged" AND "Mental disorders" OR "depression" OR "anxiety" incluindo artigos publicados de 2010 a 2020. Os resultados evidenciam que depressão e ansiedade configuram entre as desordens psiquiátricas mais comuns no período e que fatores econômicos, sociais, culturais podem influenciar tais sintomas.
\end{abstract}

Palavras-chave: Menopausa; Depressão; Climatério.

\begin{abstract}
Menopause is a period composed of several changes and transformations in the woman's life, characterizing the end of their reproductive period. Thus, the significant decrease in hormones in the female body results in a wide range of unpleasant signs and symptoms characteristic of this phase, with emphasis on psychic manifestations. The objective of the work is to carry out a bibliographic survey about the psychiatric disorders manifested during the menopause period. A search was carried out in the Pubmed, SciELO, Lilacs and Cochrane Library databases with the descriptors "Menopause" OR "Climacteric" OR "Middle Aged" AND "Mental disorders" OR "depression" OR "anxiety" including articles published in 2010 to 2020 . The results show that depression and anxiety are among the most common psychiatric disorders in the period and that economic, social and cultural factors can influence these symptoms.
\end{abstract}

Keywords: Menopause; Depression; Climacteric.

\section{Resumen}

La menopausia es um período compuesto por vários câmbios y transformaciones en la vida de las mujeres, que caracteriza el final de su período reproductivo. Así, la disminución significativa de hormonas en el cuerpo femenino da como resultado una amplia gama de signos y sintomas desagradables que son característicos de esta fase, con énfasis en las manifestaciones psiquiátricas. El objetivo del trabajo es realizar una encuesta bibliográfica sobre los transtorno psiquiátricos manifestados durante el período de la menopausia. Se realizo una busqueda en las bases de datos Pubmed, SciELO, Lilacs y Cochrane Library con los descriptores "Menopausia" O "Climaterio" Y "Transtornos Mentales" O "Depresión" O "Ansiedad" incluyendo artículos publicados de 2010 a 2020. Los resultados muestran que la depresión y la ansiedade se encuentran entre los trastornos psiquiátricos más comunes en el período y que factores económicos, sociales y culturales pueden influir en dichos sintomas.

Palabras clave: Menopausia; Depresión; Climatérico. 


\section{Introdução}

A menopausa é uma fase natural da vida da mulher, marcada por alteração hormonal e cessação da menstruação. Por volta dos 40 anos, as mulheres gradualmente começam a diminuir a produção de estrogênio. Seus períodos menstruais tornamse menos regulares e eventualmente param completamente (Potter, Schrager, Dalby, Torell, \& Hampton, 2018).

Muitas mulheres vivenciam este período de forma assintomática, ou com sintomas inexpressivos, entendendo-o como o início de uma nova etapa do amadurecimento existencial, que lhes permitirá uma vida com maior segurança e confiança (Pertesi, Coughlan, Puthusseryppady, Morris, \& Hornberger, 2019).

No entanto, mulheres podem apresentar menstruações irregulares, sintomas vasomotores, distúrbios do humor, sintomas geniturinários. Além disso, uma variedade de distúrbios psicossociais tem foi associada ao período da menopausa, incluindo depressão, nervosismo, agitação, insônia, artralgia e deficiência concentração. (Oppermann, Fuchs, Donato, Bastos \& Spritzer, 2012);(Potter, Schrager, Dalby, Torell, \& Hampton, 2018);(Baker, De Zambotti, Colrain, \& Bei, 2018);(Prairie, Wisniewski, Luther, Hess, Thurston, Wisner \& Bromberger, 2015)

Dentre as alterações mais evidentes, encontra-se os distúrbios psiquiátricos. Questões mentais e emocionais, incluindo fatores como depressão e ansiedade, intensidade dos sintomas emocionais da menopausa, sentimentos sobre o parceiro sexual, nível de autoconfiança e autoestima, imagem corporal e sentimentos de atração sexual são apresentados e discutidos .( Nazarpour, Simbar, \& Tehrani, 2016); (Stute, et al. 2020).

Tais sintomas podem tornar-se angustiantes e afetar consideravelmente os aspectos pessoais e sociais e a vida profissional das mulheres. Ressalta-se ainda que estes sintomas são mais exacerbados em mulheres que perderam seu papel social e não redefiniram seus objetivos existenciais, sendo sugerido que fatores da personalidade e tendências ansiosas prévias correlacionam-se com maior número de queixas psicológicas durante o climatério (Galvão, Farias, Azevedo, Vilar, \& Azevedo, 2007)

É evidenciado as limitações impostas nesse período, onde a forte deficiência estrogênica insatisfatoriamente no estado emocional da mesma, interferindo assim na relação familiar, na reintegração social e na adaptação sexual. Dessa forma, o objetivo deste artigo é apresentar uma revisão sistemática da literatura sobre manifestações psiquiátricas no período da menopausa.

\section{Metodologia}

Foi realizada uma revisão sistemática por meio de uma busca na literatura internacional e nacional, indexada nas seguintes bases de dados: Pubmed, SciELO, Lilacs e Cochrane Library. Os descritores utilizados foram: "Menopause” OR "Climacteric" OR "Middle Aged" AND "Mental disorders" e a busca incluiu artigos publicados em recorte atemporal. A consulta nas bases de dados foi realizada no mês de Agosto de 2020, a partir de uma leitura dos títulos e resumos capturados. Após um primeiro levantamento, os artigos pré-selecionados e aqueles sobre os quais pairassem dúvidas quanto à inclusão foram submetidos à leitura na íntegra, como nova rodada de decisões quanto à sua inclusão no estudo. Casos dúbios foram resolvidos por avaliação conjunta dos autores. Foram selecionados e incluídos na revisão estudos que elencassem manifestações/desordens psiquiátricas associadas ao período da menopausa/climatério. Não foram selecionados estudos que mencionassem tratamento farmacológico dos casos de menopausa e/ou manifestações psiquiátricas.

Após leitura de cada um dos artigos selecionados, em sua versão completa, foi empregada análise temática de conteúdo dos artigos, identificando, por meio da leitura integral de cada estudo, temas predominantes na ligação entre os objetivos, o método, a abordagem/técnica estudada e a síntese dos resultados apresentados. Em todas as etapas, as aplicações 
dos critérios de seleção dos artigos foram feitas por 3 autores e revisado por um quarto minimizando as divergências na escolha. Destaca-se que não houve troca de informações entre os autores durante a seleção.

Figura 1. Diagrama de fluxo da avaliação e seleção do artigo.

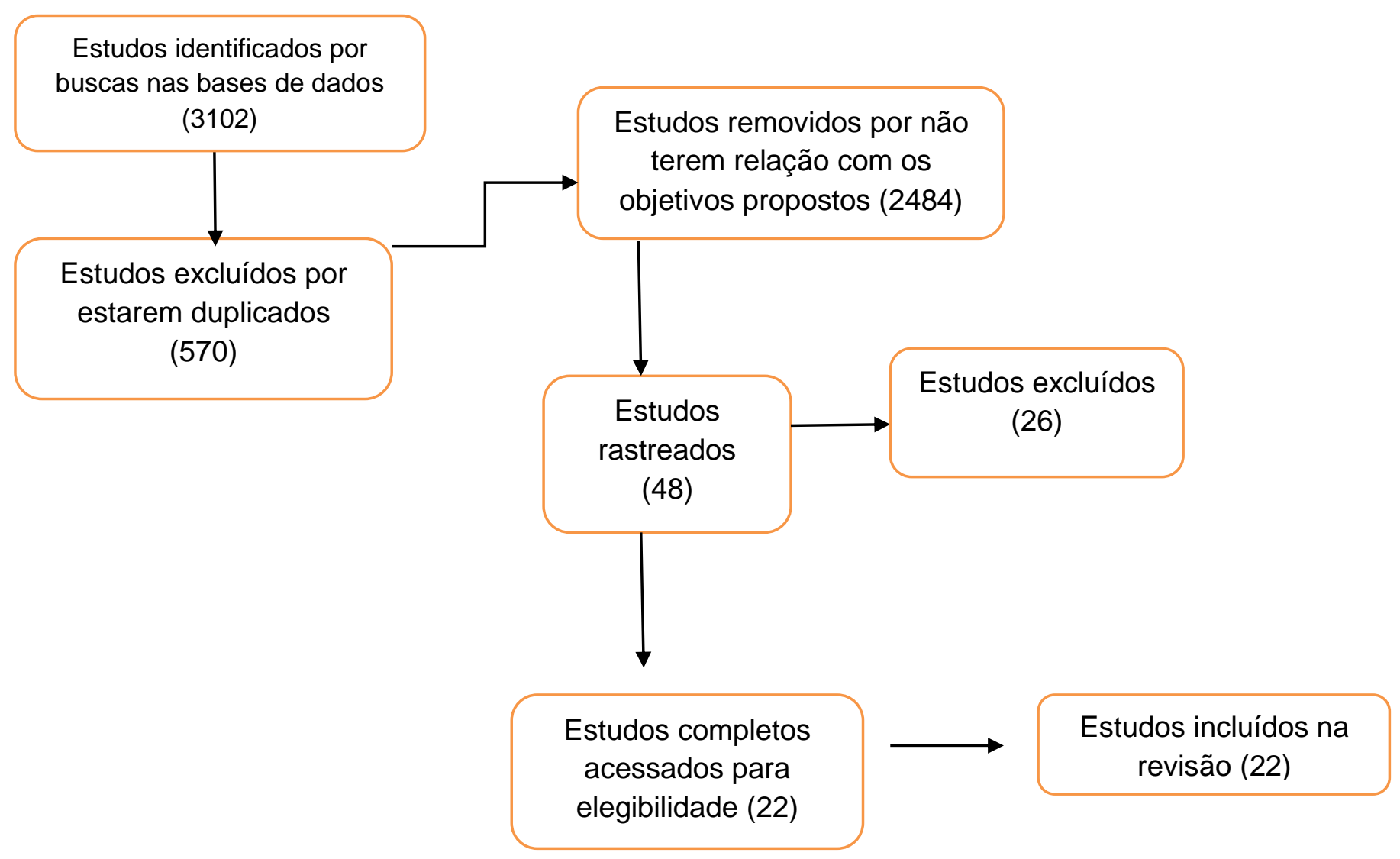

Fonte: Autores (2021).

\section{Resultados}

De todos os bancos de dados pesquisados, a pesquisa inicial gerou 3.102 resultados (Figura 1). Depois que os estudos duplicados foram removidos, 2532 artigos permaneceram. Na fase de triagem do título, 2484 artigos foram removidos da análise, restando 48 artigos para a etapa de triagem dos resumos. Na fase de triagem de resumos, 22 artigos foram considerados adequados para inclusão na triagem de texto completo (Figura 1).

A triagem de texto completo desses artigos eliminou 26, os quais não atenderam aos critérios de inclusão. Dentre as razões para a exclusão dos trabalhos elenca-se: estudos que relataram a qualidade de vida no período da menopausa, pesquisas que estudaram a ação de fármacos hormonais, estudo dos sinais de esquecimento e memória na menopausa, outros tratavam do uso de benzodiazepínicos no tratamento da ansiedade decorrente da menopausa, além das revisões da literatura. A Tabela 1 detalha os estudos que foram incluídos na síntese narrativa. 
Tabela 1: Lista dos estudos revisados que referiram manifestações psiquiátricas em mulheres no período da menopausa (n:22).

\begin{tabular}{|c|c|c|c|c|}
\hline Autor/Local & Demografia & Projeto & Instrumento de medição & Principais descobertas \\
\hline $\begin{array}{l}\text { Yanikkerem, } \\
\text { et al.,2018 } \\
\text { Turquia }\end{array}$ & $\begin{array}{l}\mathrm{N}=572 ; \\
\text { Entre } 45 \text { a } 65 \text { anos }\end{array}$ & $\begin{array}{l}\text { Quantitativo } \\
\text { transversal }\end{array}$ & $\begin{array}{ll}\cdot & \text { Inventário de Depressão de Beck } \\
& \text { (BDI) } \\
\text { · } & \text { Escala de Avaliação da } \\
\text { Menopausa (MRS) } \\
\text { · } \quad \text { Índice de Função Sexual Feminina } \\
\text { (FSFI) }\end{array}$ & $\begin{array}{l}\text { A identificação e o tratamento dos } \\
\text { problemas sexuais, emocionais e físicos no } \\
\text { período do climatério contribuem para uma } \\
\text { melhoria na qualidade de vida das } \\
\text { mulheres. }\end{array}$ \\
\hline $\begin{array}{l}\text { Slopien, et } \\
\text { al.,2017 }\end{array}$ & $\begin{array}{l}\mathrm{N}=140 \\
45 \text { mulheres na } \\
\text { transição tardia da } \\
\text { menopausa e } 95 \\
\text { mulheres na pós- } \\
\text { menopausa precoce. }\end{array}$ & $\begin{array}{l}\text { Quantitativo } \\
\text { transversal }\end{array}$ & $\begin{array}{ll}\text { - } & \text { Índice de Kupperman } \\
\text { - } & \text { Escala de Hamilton } \\
\text { - } & \text { Níveis séricos de hormônio } \\
\text { folículo-estimulante, hormônio } \\
\text { luteinizante, } 17 \beta \text {-estradiol, } \\
\text { prolactina, testosterona total, } \\
\text { sulfato de desidroepiandrosterona } \\
\text { e alopregnanolona. }\end{array}$ & $\begin{array}{l}\text { O envelhecimento reprodutivo é } \\
\text { caracterizado por uma redução dos níveis } \\
\text { circulantes de alopregnanolona que se } \\
\text { correlacionam com o índice de depressão } \\
\text { de Hamilton no início da pós-menopausa e } \\
\text { na sua transição. }\end{array}$ \\
\hline $\begin{array}{l}\text { Quiroga, et } \\
\text { al,.2017 } \\
\text { Neolonese }\end{array}$ & $\begin{array}{l}\mathrm{N}=469 \\
\text { Entre } 45 \text { e } 60 \text { anos } \\
\text { de idade }\end{array}$ & $\begin{array}{l}\text { Estudo } \\
\text { transversal não } \\
\text { experimental }\end{array}$ & $\begin{array}{ll}\text { - } & \text { Qualidade de vida específica da } \\
\text { menopausa (MENQOL) } \\
\text { - } \\
\text { Escala Hospitalar de Ansiedade e } \\
\text { Depressão (HADS) } \\
\text { · } \quad \text { Lista de verificação de adjetivo } \\
\text { para avaliação de autoconceito } \\
\text { (LAEA) } \\
\text { The Menopause Rating Scale } \\
\text { (MRS) }\end{array}$ & $\begin{array}{l}\text { O efeito moderador da qualidade de vida } \\
\text { sexual sobre a correlação previamente } \\
\text { estabelecida entre depressão e agravamento } \\
\text { dos sintomas climatéricos. }\end{array}$ \\
\hline $\begin{array}{l}\text { Chou et al., } \\
2015 \\
\text { Taiwan }\end{array}$ & $\begin{array}{l}\mathrm{N}=190 \\
\text { Idade média: } 50( \pm \\
10)\end{array}$ & $\begin{array}{l}\text { Quantitativo } \\
\text { transversal }\end{array}$ & $\begin{array}{ll}\text { - } & \text { Escala de Sintomas da Menopausa } \\
\text { - } & \text { Inventário de Cinco Fatores de } \\
& \text { Abertura de Neuroticismo } \\
\text { - } & \text { Inventário de Depressão de Ko } \\
\text { - } & \text { Entrevista diagnóstica } \\
\text { semiestruturada }\end{array}$ & $\begin{array}{l}\text { Sintomas depressivos durante a transição da } \\
\text { menopausa predizem os sintomas } \\
\text { depressivos } \\
\text { posteriores }\end{array}$ \\
\hline $\begin{array}{l}\text { Terauchi et } \\
\text { al., } 2014 \\
\text { Tokyo }\end{array}$ & $\begin{array}{l}\mathrm{N}=491 \\
\text { Idade média: } 51.8 \\
\text { 5.4) }\end{array}$ & $\begin{array}{l}\text { Quantitativo } \\
\text { transversal }\end{array}$ & $\begin{array}{l}\text { Questionário de qualidade de vida } \\
\text { relacionada à saúde na menopausa }\end{array}$ & $\begin{array}{l}\text { Os sintomas psicológicos estão presentes na } \\
\text { menopausa tanto como as dores } \\
\text { musculoesqueléticas e sintomas } \\
\text { vasomotores }\end{array}$ \\
\hline $\begin{array}{l}\text { Gibbs,et al. } \\
2013 \\
\text { Austrália }\end{array}$ & $\begin{array}{l}\mathrm{N}=66 \\
\text { Idade média: } 49,5 \\
( \pm 4,3)\end{array}$ & $\begin{array}{l}\text { Quantitativo } \\
\text { transversal }\end{array}$ & $\begin{array}{l}\text { - } \\
\text { - } \\
\text { - } \\
\text { Testes antise de protuários } \\
\text { avaliações bioquimicas e } \\
\text { hormonais }\end{array}$ & $\begin{array}{l}\text { Uma em cada três mulheres gregas } \\
\text { apresenta sintomas climatéricos moderados } \\
\text { a graves durante a transição da menopausa } \\
\text { ou nos primeiros anos da pós-menopausa. }\end{array}$ \\
\hline $\begin{array}{l}\text { Gharaibeh et } \\
\text { al, } \\
2010 \\
\text { Jordânia }\end{array}$ & $\begin{array}{l}\mathrm{N}=350 \\
\text { mulheres que } \\
\text { estavam na } \\
\text { perimenopausa ou } \\
\text { nos primeiros } 5 \text { anos } \\
\text { de pós-menopausa }\end{array}$ & $\begin{array}{l}\text { Quantitativo } \\
\text { transversal }\end{array}$ & $\begin{array}{l}\text { História sociodemográfica, } \\
\text { médica e obstétrica } \\
\text { Escala de Climatério de Greene }\end{array}$ & $\begin{array}{l}\text { Há relação significativa entre a gravidade e } \\
\text { a ocorrência dos sintomas da menopausa e a } \\
\text { idade, renda familiar, escolaridade, número } \\
\text { de filhos, percepção do estado de saúde e } \\
\text { estado menopausa. }\end{array}$ \\
\hline $\begin{array}{l}\text { T. tagen, A. } \\
\text { Mykletun, et } \\
\text { al 2008, } \\
\text { Noruega }\end{array}$ & $\begin{array}{l}\mathrm{N}=94.197 \text { mulheres, } \\
\text { entre } 35 \text { e } 60 \text { anos }\end{array}$ & Quantitativo & $\begin{array}{l}\text { Escala Hospitalar de Ansiedade e } \\
\text { Depressão (HADS,28) - } 14 \text { itens } \\
\text { da escala de LIKERT (sete para } \\
\text { ansiedade- HADS-A, e sete para } \\
\text { depressão, HADS-D) }\end{array}$ & $\begin{array}{l}\text { Pontuação significativamente maior em } \\
\text { depressão e ansiedade no período peri e } \\
\text { pós-menopausa em comparação ao período } \\
\text { pré-menopausa. }\end{array}$ \\
\hline $\begin{array}{l}\text { L.Gallichio; } \\
\text { C.Schilling, } \\
\text { et al 2007, } \\
\text { USA }\end{array}$ & $\begin{array}{l}\mathrm{N}=634 \text { mulheres, } \\
\text { entre } 45-54 \text { anos }\end{array}$ & Quantitativo & $\begin{array}{l}\text { Center for Epidemiologic Studies- } \\
\text { Depression Escala (CES-D); } \\
\text { Medição de concetrações de } \\
\text { estrôgenio e androgênio por } \\
\text { ensaio de imunoabsorção } \\
\text { enzimática } \\
\quad 4\end{array}$ & $\begin{array}{l}\text { 25\% das mulheres estavam experimentando } \\
\text { sintomas depressivos (CES-D z16); } \\
\text { Estado da menopausa e os níveis hormonais } \\
\text { não são evidentemente correlacionadas com } \\
\text { os sintomas depressivos. }\end{array}$ \\
\hline
\end{tabular}




\begin{tabular}{|c|c|c|c|c|}
\hline $\begin{array}{l}\text { J.Payne; } \\
\text { P.S.Roy et al } \\
\text { 2007, } \\
\text { Estados } \\
\text { Unidos }\end{array}$ & $\begin{array}{l}\mathrm{N}=2.412 \\
\text { Sem critérios de } \\
\text { exclusão para idade }\end{array}$ & Quantitativo & $\begin{array}{l}\text { Perguntas padronizadas sobre } \\
\text { sintomas de humor antes da } \\
\text { menstruação, dentro de um mês } \\
\text { do parto e durante perimenopausa }\end{array}$ & $\begin{array}{l}\text { 26,4\% apresentaram Transtorno de } \\
\text { Depressão e Transtorno Bipolar na } \\
\text { perimenopausa. }\end{array}$ \\
\hline $\begin{array}{l}\text { M. Amore; } \\
\text { P. Di Donato } \\
\text { et al 2007, } \\
\text { Itália }\end{array}$ & $\mathrm{N}=4.073$ mulheres & $\begin{array}{l}\text { Pesquisa postal } \\
\text { transversal }\end{array}$ & $\begin{array}{l}\text { Questionário de Saúde da Mulher } \\
\text { (WHQ) } \\
\text { Arquivo pessoal com: status } \\
\text { social, nível cultural, } \\
\text { características da família, ciclos } \\
\text { menstruais recentes, história } \\
\text { ginecológica e operações, } \\
\text { suposição de drogras, eventos de } \\
\text { vida no último ano e depressão } \\
\text { vitalícia }\end{array}$ & $\begin{array}{l}\text { Os sintomas depressivos e sexuais } \\
\text { apresentaram maior gravidade no grupo } \\
\text { pós-menopausa. }\end{array}$ \\
\hline $\begin{array}{l}\text { C. Travers; } \\
\text { S. M. } \\
\text { O’Neill et al } \\
2005 \text {, } \\
\text { Austrália }\end{array}$ & $\begin{array}{l}\mathrm{N}=500 \text { mulheres, } \\
\text { entre } 40-80 \text { anos }\end{array}$ & $\begin{array}{l}\text { Estudo } \\
\text { Longitudinal- } \\
\text { Coorte }\end{array}$ & $\begin{array}{l}\text { Escala Climatérica de Greene e } \\
\text { informação sobre o estado da } \\
\text { menopausa e uso de TRH por } \\
\text { meio de entrevista clínica por } \\
\text { médico }\end{array}$ & $\begin{array}{l}\text { Faixa etária de } 50-59 \text { anos teve pontuações } \\
\text { mais altas em sintomas vasomotores e } \\
\text { depressivos em comparação as outras } \\
\text { faixas etárias. }\end{array}$ \\
\hline $\begin{array}{l}\text { N. Anviz; D. } \\
\text { Brambilla et } \\
\text { al 1994, } \\
\text { Massachusse } \\
\text { tts }\end{array}$ & $\begin{array}{l}\mathrm{N}=2.565 \text { mulheres, } \\
\text { entre } 45-55 \text { anos }\end{array}$ & $\begin{array}{l}\text { Quantitativo, } \\
\text { estudo } \\
\text { longitudinal }\end{array}$ & $\begin{array}{l}\text { Escala do Centro de Estudos } \\
\text { Epidemiológicos (CES-D) }\end{array}$ & $\begin{array}{l}\text { A vivência de um longo período de } \\
\text { perimenopausa (pelo menos } 27 \text { meses) foi } \\
\text { associado ao aumento do risco de } \\
\text { depressão, explicado pelo aumento dos } \\
\text { sintomas da menopausa e não pelo próprios } \\
\text { status da menopausa. }\end{array}$ \\
\hline $\begin{array}{l}\text { Chedraui, et } \\
\text { al,.2007 } \\
\text { Equador }\end{array}$ & $\begin{array}{l}\mathrm{N}=300 \\
\text { A idade média foi de } \\
45,1+/-3,1 \text { anos } \\
\text { (mediana 45). }\end{array}$ & $\begin{array}{l}\text { Quantitativo } \\
\text { transversal }\end{array}$ & $\begin{array}{l}\text { - The Menopause Rating Scale } \\
\text { (MRS). }\end{array}$ & $\begin{array}{l}\text { A idade, a menopausa, a inatividade sexual } \\
\text { e o nível de escolaridade foram fatores de } \\
\text { risco independentes preditores de sintomas } \\
\text { menopáusicos mais graves. }\end{array}$ \\
\hline $\begin{array}{l}\text { Binfa, et } \\
\text { al,.2004 } \\
\text { Chile }\end{array}$ & $\begin{array}{l}\mathrm{N}=300 \\
\text { Entre } 40 \text { e } 59 \text { anos } \\
\text { de idade }\end{array}$ & $\begin{array}{l}\text { Quantitativo } \\
\text { transversal }\end{array}$ & $\begin{array}{ll}\text { - } & \text { Greene (sintomas climatéricos), } \\
\text { - } & \text { Cooper (sintomas psicossomáticos } \\
\text { de estresse), } \\
\text { - } \quad \text { Smilkstein (disfunção familiar), } \\
\text { - } \quad \text { Duke-UNC (apoio social) } \\
\text { - } \quad \text { Israel (eventos de vida) } \\
\end{array}$ & $\begin{array}{l}\text { Sintomas climatéricos que surgem na } \\
\text { perimenopausa são mais intensos nas } \\
\text { mulheres com predisposição biológica, } \\
\text { como a síndrome pré-menstrual, e são } \\
\text { modulados por fatores psicossociais. }\end{array}$ \\
\hline $\begin{array}{l}\text { Nülüfer } \\
\text { Erbil,.2017 } \\
\text { Turquia }\end{array}$ & $\mathrm{N}=109$ & $\begin{array}{l}\text { Estudo descritivo } \\
\text { e transversal }\end{array}$ & $\begin{array}{ll}\text { - } & \text { Escala de Atitude Frente à } \\
\text { Menopausa (ATMS), } \\
\text { - } \quad \text { Escala de Imagem Corporal (BIS) } \\
\text { - Inventário de Depressão de } \\
\text { Beck (BDI). }\end{array}$ & $\begin{array}{l}\text { Mulheres com atitude otimista em relação à } \\
\text { menopausa tendem a ter uma imagem } \\
\text { corporal mais positiva e seu nível de } \\
\text { depressão é menor }\end{array}$ \\
\hline $\begin{array}{l}\mathrm{Li} \text {, et } \\
\text { al,.2008 } \\
\text { Pequim }\end{array}$ & $\begin{array}{l}\mathrm{N}=1280 \\
\text { Idades entre } 45-59 \\
\text { anos de idade. }\end{array}$ & $\begin{array}{l}\text { Quantitativo } \\
\text { transversal }\end{array}$ & $\begin{array}{l}\text { - Informações gerais: incluindo } \\
\text { características sociais, familiares e } \\
\text { situação econômica, menstruação } \\
\text { e informações de parto, sintomas } \\
\text { climatéricos e evento negativo em } \\
\text { um ano recente. } \\
\text { Escala de classificação de suporte } \\
\text { social: } \\
\text { Escala de Autoavaliação de } \\
\text { Depressão de Zung (SDS } \\
\text { Escala de Autoavaliação de } \\
\text { Ansiedade (SAS) de Zung } \\
\end{array}$ & $\begin{array}{l}\text { Depressão e ansiedade foram sintomas } \\
\text { comuns em mulheres chinesas durante a } \\
\text { transição e pós-menopausa. Alguns fatores } \\
\text { psicossociais podem desempenhar um papel } \\
\text { na prevalência deles. }\end{array}$ \\
\hline $\begin{array}{l}\text { Carolina } \\
\text { Correia } \\
\text { Tomás, et al. } \\
\text { 2018 Lisboa }\end{array}$ & $\begin{array}{l}\mathrm{N}=10 \\
\text { Idades entre } 43 \text { e } 86 \\
\text { anos }\end{array}$ & $\begin{array}{l}\text { Quantitativo } \\
\text { tranversal }\end{array}$ & $\begin{array}{l}\text { Entrevista semiestruturada } \\
\text { Análise de contéudo e temática da } \\
\text { entrevista feita por dois } \\
\text { avaliadores de forma dependente } \\
\text { com recurso ao MAXQDA, } \\
\text { seguida por uma análise de } \\
\text { frequência. }\end{array}$ & $\begin{array}{l}\text { A cessação de menstruação e os sintomas } \\
\text { vasomotores foram as representações da } \\
\text { menopausa mais frequentes ( } 50 \%) \text {, sendo a } \\
\text { primeira a consequência positiva mais } \\
\text { mencionada }(50 \%) \text { e a segunda a negativa } \\
(40 \%) \text {. Quanto às representações de } \\
\text { andropausa, as respostas mais dadas foram } \\
\text { associadas ao envelhecimento, variações na } \\
\text { líbido, perda de capacidades e a visão da } \\
\text { andropausa como um processo equivalente } \\
\text { à menopausa ( } 30 \%) \text {. A depressão foi } \\
\text { identificada como a consequência negativa }\end{array}$ \\
\hline
\end{tabular}




\begin{tabular}{|c|c|c|c|c|}
\hline & & & & mais referida $(30 \%)$ \\
\hline $\begin{array}{l}\text { Adrián } \\
\text { Enrique } \\
\text { Hernández- } \\
\text { Muñoz, et al. } \\
2019 . \\
\text { Guadalajara, } \\
\text { Jalisco }\end{array}$ & $\begin{array}{l}\mathrm{N}=252 \text { mulheres } \\
\text { Em início da } \\
\text { menopausa }(48 \pm \\
1,7 \text { anos })\end{array}$ & $\begin{array}{l}\text { Analítico } \\
\text { Transversal }\end{array}$ & $\begin{array}{l}\text { - } \quad \text { Inventário de Depressão de Beck } \\
\text { - } \quad \text { Avaliação da Menopausa } \\
\text { - Análises descritivas e de } \\
\text { associação realizadas por Odds } \\
\text { Ratio }\end{array}$ & $\begin{array}{l}\text { A prevalência de TDM foi de } 40,5 \% \text {, tendo } \\
\text { associação com o histórico de uso de } \\
\text { contraceptivos e com o agravamento dos } \\
\text { sintomas da menopausa. A análise ajustada } \\
\text { determinou que } \\
\text { há maior risco de apresentar TDM quando } \\
\text { há alterações nos domínios } \\
\text { sintomatológicos, como em } \\
\text { somático (OR 3,96, IC 95\% 1,58-9,95), } \\
\text { urogenital (OR 4,29, IC 95\% 2,13-8,65) e } \\
\text { psicológico (OR 13,55, } \\
\text { IC de 95\% 3,97-46,30). }\end{array}$ \\
\hline $\begin{array}{l}\text { Freeman, et } \\
\text { al., } 2006 \\
\text { Pensilvânia }\end{array}$ & $\begin{array}{l}\mathrm{N}=436 \text { mulheres, } \\
\text { dessas } 231 \text { sem } \\
\text { histórico de } \\
\text { depressão }\end{array}$ & $\begin{array}{l}\text { Estudo } \\
\text { longitudinal }\end{array}$ & $\begin{array}{l}\text { Escala do centro de estudos } \\
\text { epidemiológicos da depressão ( } \\
\text { CES/D) } \\
\text { - Avaliação dos transtornos mentais } \\
\text { da atenção básica ( PRIME-MD) }\end{array}$ & $\begin{array}{l}\text { Transição para a menopausa e suas } \\
\text { mudanças do meio hormonal estão } \\
\text { fortemente associados ao novo início de } \\
\text { humor deprimido entre mulheres sem } \\
\text { histórico de depressão }\end{array}$ \\
\hline $\begin{array}{l}\text { Veras, et } \\
\text { al.,2006 Rio } \\
\text { de Janeiro }\end{array}$ & $\begin{array}{l}\mathrm{N}=86 \text { mulheres que } \\
\text { encontravam-se em } \\
\text { tratamento no } \\
\text { ambulatório de } \\
\text { menopausa do } \\
\text { Instituto de } \\
\text { Ginecologia da } \\
\text { Universidade } \\
\text { Federal do Rio de } \\
\text { Janeiro }\end{array}$ & $\begin{array}{l}\text { Quantitativo } \\
\text { transversal }\end{array}$ & $\begin{array}{l}\text { Mini International } \\
\text { Neuropsychiatric } \\
\text { Interview (MINI), }\end{array}$ & $\begin{array}{l}\text { Alta prevalência de transtornos mentais } \\
\text { entre mulheres em atendimento } \\
\text { ambulatorial na menopausa além de uma } \\
\text { alta taxa de prevalência de comorbidades } \\
\text { complicadoras do transtorno primário }\end{array}$ \\
\hline $\begin{array}{l}\text { Pereira, et } \\
\text { al., } 2009 \text { São } \\
\text { Paulo }\end{array}$ & $\begin{array}{l}\text { N=875 mulheres na } \\
\text { transição } \\
\text { menopausal e pós- } \\
\text { menopausa, } \\
\text { cadastradas no } \\
\text { Programa de Saúde } \\
\text { da Família (PSF) }\end{array}$ & $\begin{array}{l}\text { Estudo } \\
\text { observacional } \\
\text { transversal }\end{array}$ & $\begin{array}{l}\text { Inventário de Ansiedade de BECK } \\
\text { (BAI) } \\
\text { Questionário composto de dados } \\
\text { pessoais, socioeconômicos, } \\
\text { história ginecológica, morbidade, } \\
\text { hábitos de vida, atividade física e } \\
\text { ansiedade. }\end{array}$ & $\begin{array}{l}\text { A ansiedade foi muito prevalente, atingindo } \\
\text { quase metade da população estudada e os } \\
\text { principais fatores associados à ansiedade } \\
\text { foram os hábitos de vida e as condições } \\
\text { socioeconômicas. }\end{array}$ \\
\hline
\end{tabular}

\section{Discussão}

Fonte: Autores (2021).

Aproximar-se da meia-idade geralmente traz mais estresse, ansiedade e medo. Isso pode ser parcialmente atribuído a mudanças físicas, como a diminuição dos níveis de estrogênio e progesterona. Ondas de calor, suor e outros sintomas da menopausa podem causar interrupções. Também pode haver mudanças emocionais, como preocupações com o envelhecimento, perda de familiares ou filhos saindo de casa. Para algumas mulheres, a menopausa pode ser um período de isolamento ou frustração. A família e os amigos nem sempre entendem o que você está passando ou dão o apoio de que você precisa. Somando-se a isso, o humor depressivo e as manifestações de natureza psiquiátrica parecem ser os sintomas mais comuns experimentados durante a menopausa.

No Brasil, a perspectiva de vida é em torno dos 72,4 anos e um terço da vida das mulheres será vivido no climatério, estimando-se que 33\% das mesmas sofrerão pelo menos um episódio de depressão durante a vida, com prevalência de $9 \%$ no período do climatério (Gibbs, Lee \& Kulkami 2013).

As alterações hormonais que ocorrem no climatério poderiam, em mulheres predispostas, desencadear sintomas ansiosos, através de mecanismo idêntico ao da ocorrência da síndrome de tensão pré-menstrual (Payne, Roy, Murphy-Fherenz, Weishman, Swartz, McInnis \& Potash 2007). Ademais, foi evidenciado em um estudo que mulheres com uma atitude mais positiva sobre esse período tendem a ter uma aceitação corporal maior e consequentemente um nível e gravidade de depressão menor (Gharaibeh, Al-Obeisant \& Hattab 2010). 
Estudos relacionaram o estilo de vida das mulheres nesse período, associados aos efeitos impostos, analisaram quantitativamente os sintomas depressivos em mulheres climatéricas em diferentes lugares, encontrando, $16 \%$ nos Estados Unidos, 40,2\% na Espanha e 37,6\% no Peru, demonstrando uma alta incidência dos efeitos negativos na qualidade de vida das mulheres inseridas no período da menopausa (Yanikkerem, Göker, Çakır \& Esmeray 2018).

Os maiores riscos para depressão em mulheres de meia idade podem estar no período de transição para a menopausa, ao invés do período de pós-menopausa. Aliado a isso é evidenciado que, indivíduos que experimentaram sintomas depressivos exacerbados antes da menopausa têm uma maior probabilidade no aumento de reações menopausais, sintomas vasomotores adicionais ou ter uma história de depressão durante o período da menopausa (Quiroga, Larroy \& González-Castro 2017).

Diante do resultado exposto do estudo supracitado, é evidenciado a ocorrência dos efeitos desestabilizadores das flutuações cíclicas do estradiol que aumentam gradativamente ao envelhecimento ovariano, particularmente na transição para menopausa, tornam-se, de fato, um fator importante para o desenvolvimento de sintomas depressivos, assim como, para o próprio diagnóstico do transtorno psíquico.

Foi evidenciado ainda, que a vivência da perimenopausa a longo prazo (pelo menos 27 meses) foi associado a um aumento do risco de depressão. Esse fato é explicado pelo aumento dos sintomas do período da menopausa e não pelo status próprio da menopausa, (Quiroga, Larroy \& González-Castro 2017), sendo definida a gravidade desse período climatérico, mais especificamente no pós menopausa, principalmente, os sintomas depressivos e sintomas sexuais (Trayers, O’neill, King, Battistutta \& Khoo 2005).

Além de que, mulheres com Transtorno Depressivo Maior (TDM) são associadas significativamente a ocorrência de sintomas depressivos durante o período de transição da menopausa, bem como os sintomas depressivos após 30 meses, sendo explicado pelo fato dos hormônios reprodutivos estarem em associação com o humor em indivíduos com histórico de depressão, comparado com mulheres sem histórico, como, aumento de hormônio folículos estimulante (FSH) e hormônio luteinizante (LH) e diminuição dos níveis de inibina B e o aumento da variabilidade de FSH e estradiol tem sido significativamente correlacionado com a ocorrência de depressão entre mulheres com TDM (Chou, Ko, Wu, Chang \& Tung 2015).

Já em relação a faixa etária, foi visto uma prevalência entre 50-59 anos, encontrada pontuações mais altas em sintomas vasomotores e depressivos em comparação as outras faixas etárias, na qual, somada ainda ao período em si, a inatividade sexual e o nível de escolaridade, tornam-se fatores de riscos independentes preditores de sintomas de menopausa mais graves (Estrela et al 2018); (Pereira et al 2018). As temáticas psicológicas abordadas nos trabalhos são diversas, destacando-se qualidade de vida e impacto da doença nos diversos âmbitos. A abordagem multidisciplinar que poderia ser oferecido a mulheres durante este período seria reduzir os sintomas físicos e psicológicos, portanto, a qualidade de vida das mulheres seria, de fato, maior.

Os profissionais de saúde devem abordar as mulheres em seus problemas sexuais e orientá-las para o tratamento adequadamente sobre sua condição (Yanikkerem, Goker, Cakur \& Esmeray 2018). Porém, sabe- se que os sintomas típicos do climatério também podem estar associados a doenças subjacentes, potencializando assim o papel do profissional em analisar a saúde em geral da mulher investigando doenças secundárias e não apenas confiando no diagnóstico prévio da menopausa (Gharaibeh, Al-Obeisat \& Hattab 2010).

\section{Conclusão}

Informações acerca de desordens psiquiátricas durante o período da menopausa são de suma importância para que estratégias de tratamento e intervenção sejam feitas durante ou antes do período em destaque. Evidências mostram que o 
período menopausal manifesta-se de diferentes formas no organismo feminino, sendo que este responde de maneiras diferentes relacionando-se com idade e fator econômico.

\section{Referências}

Potter, B., Schrager, S., Dalby, J., Torell, E., \& Hampton, A. (2018). Primary care—Clinics in office practice. Menopause, 45(4), 625-641.

Baker, F. C., De Zambotti, M., Colrain, I. M., \& Bei, B. (2018). Sleep problems during the menopausal transition: prevalence, impact, and management challenges. Nature and science of sleep, 10, 73.

Prairie, B. A., Wisniewski, S. R., Luther, J., Hess, R., Thurston, R. C., Wisner, K. L., \& Bromberger, J. T. (2015). Symptoms of depressed mood, disturbed sleep, and sexual problems in midlife women: cross-sectional data from the Study of Women's Health Across the Nation. Journal of women's health, 24(2), $119-126$.

Monteleone, P., Mascagni, G., Giannini, A., Genazzani, A. R., \& Simoncini, T. (2018). Symptoms of menopause-global prevalence, physiology and implications. Nature Reviews Endocrinology, 14(4), 199-215.

Zhu, D., Chung, H. F., Dobson, A. J., Pandeya, N., Brunner, E. J., Kuh, D., ... \& Mishra, G. D. (2020). Type of menopause, age of menopause and variations in the risk of incident cardiovascular disease: pooled analysis of individual data from 10 international studies. Human Reproduction, 35 (8), $1933-1943$.

Riecher-Rossler, A., \& De Geyter, C. (2007). O futuro papel do tratamento com estrogênios na saúde mental. Swiss Medical Weekly, 137 (41-42), 565-572.

Nazarpour, S., Simbar, M., \& Tehrani, F. R. (2016). Factors affecting sexual function in menopause: A review article. Taiwanese Journal of Obstetrics and Gynecology, 55(4), 480-487.

Oppermann, K., Fuchs, S. C., Donato, G., Bastos, C. A., \& Spritzer, P. M. (2012). Physical, psychological, and menopause-related symptoms and minor psychiatric disorders in a community-based sample of Brazilian premenopausal, perimenopausal, and postmenopausal women. Menopause, 19(3), 355-360.

Galvão, L. L. L. F., Farias, M. C. S., Azevedo, P. R. M. D., Vilar, M. J. P., \& Azevedo, G. D. D. (2007). Prevalência de transtornos mentais comuns e avaliação da qualidade de vida no climatério. Revista da Associação Médica Brasileira, 53, 414-420.

Pertesi, S., Coughlan, G., Puthusseryppady, V., Morris, E., \& Hornberger, M. (2019). Menopause, cognition and dementia-A review. Post reproductive health, 25(4), 200-206.

Stute, P., Spyropoulou, A., Karageorgiou, V., Cano, A., Bitzer, J., Ceausu, I., ... \& Lambrinoudaki, I. (2020). Management of depressive symptoms in peri-and postmenopausal women: EMAS position statement. Maturitas, 131, 91-101.

Truong, D., \& Marsh, W. (2019). Bipolar Disorder in the Menopausal Transition. Current psychiatry reports, 21(12), 1-7.

Yanikkerem, E., Göker, A., Çakır, Ö., \& Esmeray, N. (2018). Effects of physical and depressive symptoms on the sexual life of Turkish women in the climacteric period. Climacteric, 21(2), 160-166.

Slopien, R., Pluchino, N., Warenik-Szymankiewicz, A., Sajdak, S., Luisi, M., Drakopoulos, P., \& Genazzani, A. R. (2018). Correlation between allopregnanolone levels and depressive symptoms during late menopausal transition and early postmenopause. Gynecological Endocrinology, $34(2), 144-147$.

Quiroga, A., Larroy, C., \& González-Castro, P. (2017). Climacteric symptoms and their relation to feminine self-concept. Climacteric, 20(3), $274-279$.

Chou, C. H., Ko, H. C., Wu, J. Y. W., Chang, F. M., \& Tung, Y. Y. (2015). Effect of previous diagnoses of depression, menopause status, vasomotor symptoms, and neuroticism on depressive symptoms among climacteric women: A 30-month follow-up. Taiwanese Journal of Obstetrics and Gynecology, 54(4), 385-389.

Gibbs, Z., Lee, S., \& Kulkarni, J. (2013). Factors associated with depression during the perimenopausal transition. Women's Health Issues, 23(5), e301-e307. Gharaibeh, M., Al-Obeisat, S., \& Hattab, J. (2010). Severity of menopausal symptoms of Jordanian women. Climacteric, 13(4), 385-394.

Tangen, T., \& Mykletun, A. (2008). Depression and anxiety through the climacteric period: an epidemiological study (HUNT-II). Journal of Psychosomatic Obstetrics \& Gynecology, 29(2), 125-131.

GALLICCHIO, Lisa et al. Correlates of depressive symptoms among women undergoing the menopausal transition. Journal of psychosomatic research, v. 63, n. 3, p. 263-268, 2007.

Payne, J. L., Roy, P. S., Murphy-Eberenz, K., Weismann, M. M., Swartz, K. L., McInnis, M. G., ... \& Potash, J. B. (2007). Reproductive cycle-associated mood symptoms in women with major depression and bipolar disorder. Journal of affective disorders, 99(1-3), 221-229.

Amore, M., Di Donato, P., Berti, A., Palareti, A., Chirico, C., Papalini, A., \& Zucchini, S. (2007). Sexual and psychological symptoms in the climacteric years. Maturitas, 56(3), 303-311.

Travers, C., O'neill, S. M., King, R., Battistutta, D., \& Khoo, S. K. (2005). Greene Climacteric Scale: norms in an Australian population in relation to age and menopausal status. Climacteric, $8(1), 56-62$.

Estrela, C. (2018). Metodologia Científica: Ciência, Ensino, Pesquisa. Editora Artes Médicas.

Pereira A. S. et al. (2018). Metodologia da pesquisa científica. [free e-book]. Santa Maria/RS. Ed. UAB/NTE/UFSM. 